\title{
Improved Data Security for Storing and Authenticating in Cloud using ERSA Algorithm
}

\author{
Rokesh Kumar Yarava, J Kavitha
}

\begin{abstract}
Present days, huge amount of data stored with cloud service providers. The Third-party auditors (TPAs), with support of cryptography, are frequently utilized to prove this data. Auditing will be capability for cloud clients to prove the existence \&functioning of their supplier's security measures. Authentication is done by using username and password. The important point in authentication is to protect data from the access of unauthorized people. The proposed scheme is Enhanced RSA (ERSA) Algorithm. This paper presents solution to enhance the security and privacy to stored data in cloud. Result demonstrates that this scheme can progress the security of data that stored in cloud.
\end{abstract}

Index Terms - cloud computing (CC); information security; confidentiality; integrity, Enhanced RSA (ERSA) algorithm

\section{INTRODUCTION}

The cloud computing will be an administration delivering method on internet. It could offer clients with versatile benefits as needed through the web \&generally distinguished $\&$ applied. To use the resources of computing are very securely and effectively, people start to pay attention to unseen safety issues in cloud. The access control will be very significant dimensions to ensure the cloud computing safety. The environment of $\mathrm{CC}$ will be usual disseminated environment; consequently the anonymity, dynamism, \&distribution of data services \&assets have notable characteristics of $\mathrm{CC}$ environment [1].

The main technique utilized in cryptography may be encryption. This technique conserves integrity \& confidentiality of information. The confidentiality characteristic will be information constantly produced available to approved gatherings. The integrity is not permitting unapproved gathering to change the information that will be saved by client. The access control is the main significant dimension to ensure the safety of $\mathrm{CC}$, numerous clients are using this access control to their data is confidential [2].

Cloud is also permits the similar strategy towards the concern of security. As stated by cloud environment, encrypting the information of clients might be finished by 2 parts, they are CSP or TPA. ${ }^{\mathrm{T}}$ he 2 elementary purposes for development in storage of data in clouds are cloud based storage framework recoveries clients the overhead connected with assets that might make fundamental for an accepted in-house information storage result, \& in its place, offloads these

Revised Manuscript Received on October 22, 2019.

Rokesh Kumar yarava, Assistant Professor, Dept. of CSE, Malla Reddy Engineering college (Autonomous), Hyderabad, India.

J Kavitha, Assistant Professor, Dept. of CSE, Malla Reddy Engineering college (Autonomous), Hyderabad, India.

overheads to cloud, and ability proliferation compelled personal versatile registering devices [3].

Therefore, in present days the clients become under the encrypting methodology their sensitive information before sending it to cloud for storage. The ERSA technology utilizes 2 extra prime numbers in standard RSA method. This thought is raised from security RSA \& high speed method that utilized 2 random numbers to generation procedure of key. Using this proposed algorithms proposed method insures the confidentiality, integrity, availability and durability property of service which will improve the cloud services reliability [5].

This idea is raised from Security RSA \&High Speed method that utilized 2 random numbers for key generation procedure. Using this proposed algorithms proposed method insures the confidentiality, integrity, availability and durability property of service which will improve the cloud services reliability.

\section{BACKGROUND}

The public framework of auditing the data safely stored in CC $\&$ gives a PPA protocol. It empowers an outside auditor to audit cloud information of clients without know the information. A productive remote information auditing technique to storage the safety information in CC. Furthermore to dynamic operation, $3^{\text {rd }}$ party verification, \&other functions [1].

The TCM is presented under control method. The common trust among cloud service nodes \& clients have guaranteed by trust device. The trust clients are access to cloud; concurrently clients might choose reliable service nodes of cloud. The method had a best malicious detection capability $\&$ adaptability. In environment of $\mathrm{CC}$, whereas the reliability \& safety of both connection parties have ensured, after the safety of information might be efficiently connections among cloud \& clients [2].

The storage frameworks with various information sources are very applicable in numerous real world situations. The CSSP preserves a cloud storage giving the essential structure to make, store \&upgrade outsourced databases, \&makes this information accessible to subscribing customers. The customers are provided for read-only access to data base\& commissioned approved customers have permitted to make variances under database [3].

In DCS supplier has infinite access to stored information, \& this perspective turns into particularly critical whereas hybrid or public clouds have utilized. 


\section{Improved Data Security for Storing and Authenticating in Cloud using ERSA Algorithm}

The data stored in CC will be secured from hardware faults. Whereas utilizing the CC \& DCS in specific, there arises few problems associated to integrity \& confidentiality of data in procedure of transmission \& storage [4].

In RSA, an asymmetric key technology utilizing 2 diverse keys to decryption \& encryption procedures. The size of key might be differed to create the procedure of encryption is strong. Consequently, it will be critical to the attackers to interrupt the information. Expanding key size correspondingly increments the time taken for procedure of decryption \& encryption [5].

This paper introduces an enhanced data auditing and authentication scheme which helps to improve privacy of data owners this proposed theory.

\section{Previous WORK DONE}

The work [1] recommended a grid-based charging \& joint routing technology for industrial remote rechargeable sensor networks. The authors Tie Qiuet. All recommended a greedy method with little world to enhancing the robustness for heterogeneous IoT. The suggested schema utilizes a best load dissemination approach that significantly lessens the computational overhead of customer. The recommended plan incorporates a lapse reaction scheme, \& outcomes indicate that result has better error-handling capacity \& provides lesser overhead payments for communication \& computation than other methodologies.

The work [2] suggested trust based access control strategy in multi-domain of CC. The suggested strategy joining with TM, MTBAC method. The MTBAC method take credibility of cloud service nodes \& behavior trust of clients under deliberation. The relationships of trust among service nodes of cloud \& clients have recognized towards mutual trust component. The access control safety issues have resolved by executing MTBAC method under CC environment. The results represents that MTBAC method might ensure the collaboration among cloud service nodes \& clients.

The work [3] suggested Auditing for information reliability \& integrity in cloud storage. The suggested model introduces a scheme of query authentication for cloud-based storage framework whereas information will be retrieved towards customers \& populated towards numerous sources. The framework permits customers to confirm the integrity \& authenticity of retrieved information in proficient \& scalable way, without need of implicit trust on storage service supplier. The suggested method will be rely on lately suggested "multi-trapdoor hash functions", utilizing its assets to accomplish neighbor consistent computation \&correspondence overhead to validating inquiry responses, in any case of the information size, or the amount of sources. The work [5] recommended system for upgrading the information safety in cloud toward executing hybrid (Rsa\&Aes) encryption method. The encryption may be completed by utilizing 1 popular asymmetric or symmetric key method like DES, AES, Blowfish, DES, RSA, \& triple DES and so on; RSA method is a asymmetric key method utilizing 2 diverse keys for decryption \& encryption procedures. The size of key might be varied to make encryption procedure will be strong. Therefore, it will be critical for attackers to interrupt the information. Expanding the size of key consistently enhances the time for decryption $\&$ encryption procedure. The suggested method lessens the time for decryption \&encryption procedures by separating the document under pieces \& increases the quality of method by expanding the size of key. This quality paves the path to store the information in cloud towards the clients without any trouble.

\section{EXISTINGMETHODOLOGIES}

4.1 An Efficient Protocol with Bidirectional Verification The suggested auditing protocol helps dynamic information operations that will be proficient\& analyzed to safe in random oracle method. The suggested auditing protocol gives the help for statistical examination \& bidirectional confirmation. The public framework of auditing the safety of information stored in CC \& offer PPA protocol.

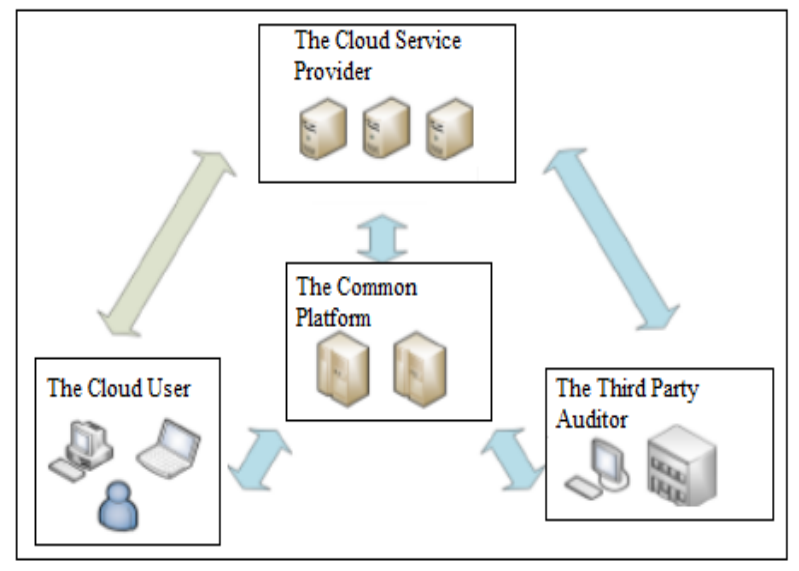

Fig. 1, System model

The recommended plan empowers an outside auditor to audit clients cloud information without learning the information. To make preparing much efficient, the calculations of customer account for little portion of total computation. Whereas the document will be changed, the owner of information might check to find if significant portions have in better situation. Further, the information manager might define the area of error, thus securing the information, which not been transformed.

\section{4.2 A Mutual Trust BASEd ACCess Control MODEL}

The recommended system is presented trust computation method under access control method. The mutual trust among cloud service nodes \& clients have ensured by trust mechanism. The MTBAC method not recognizes attitude of client trust \& ensure the access of clients request postures no malevolent risk to cloud server, \& takes cloud administration node's validity under account. 


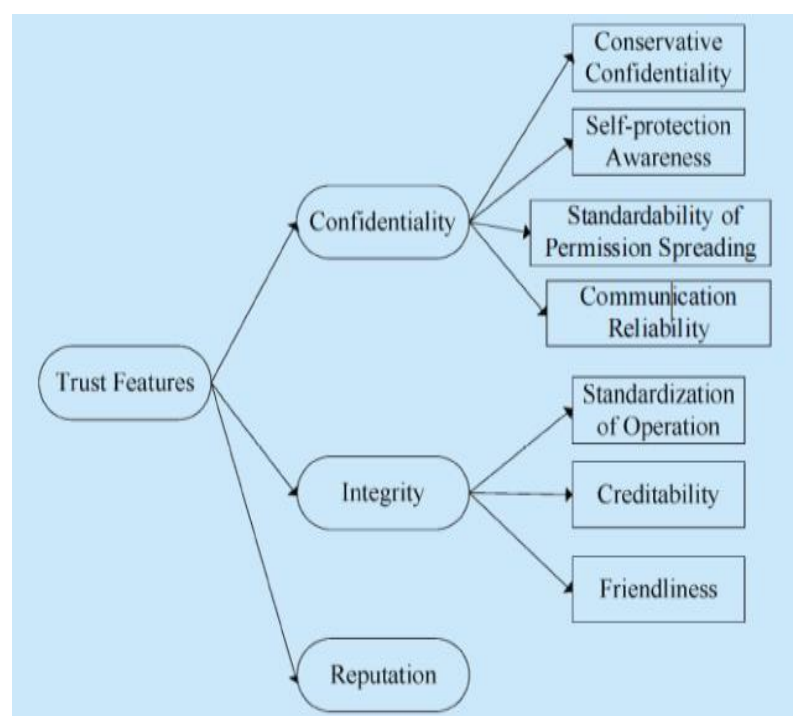

Fig. 2, the Division of Trust Attribute

The mutual trust method of cloud service nodes \& clients has 2 part construction. One portion will be trust evaluation method of client's attitude \& another portion will be trust computation method by cloud service nodes. The network level, made dependent upon of 3 attributes, reputation, confidentiality, \& integrity.

\section{4.3 EFFICIENT AND SCALABLE QUERY AUTHENTICATION WITH MULTIPLE DATA SOURCES}

The recommended system introduces a new component for query verification in cloud-based storage framework with help for several sources, which accomplishes persistent computation \& correspondence overheads in any case of size of information, or amount of sources. The suggested plan accomplishes this towards producing confirmation tags of individual information components \& aggregating tags of different information components utilizing a new system dependent on late suggested standard named as multitrapdoor hashing plan.

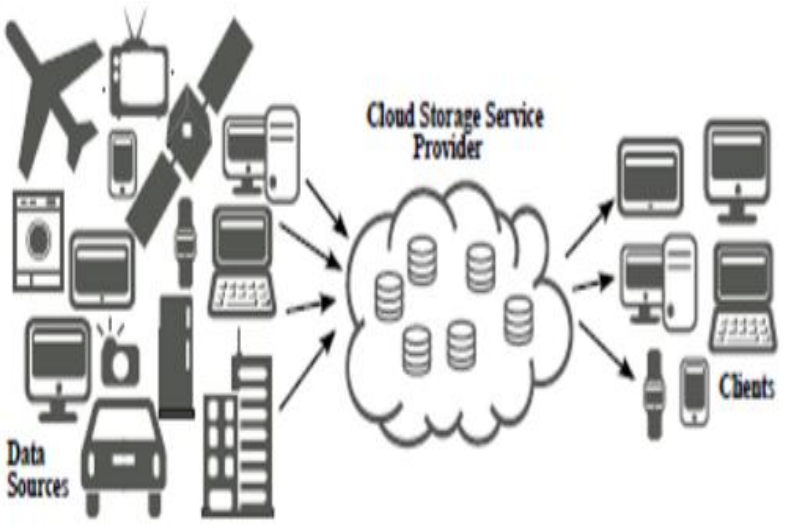

Figure.3. Cloud based storage system

The query authentication method for multi-source cloud based storage frameworks to give high safety \& light weight operation. In terms of cost of operation, goal will be to decrease the communication, computation, \& storage overhead at every entity utilizing cryptographic method, which scales with numerous sources, databases size \& response of query.

\section{4.4 THE METHOD OF ENSURING CONFIDENTIALITY AND INTEGRITY DATA}

A method that describes the use of separate services outside the cloud for authentication, data management and metadata storage to eliminate the possibility of obtaining unauthorized access to data, and the use of metadata to perform integrity control. The owner of the database limits the access to data that is stored in an encrypted form and does not allow provider to interact with database.

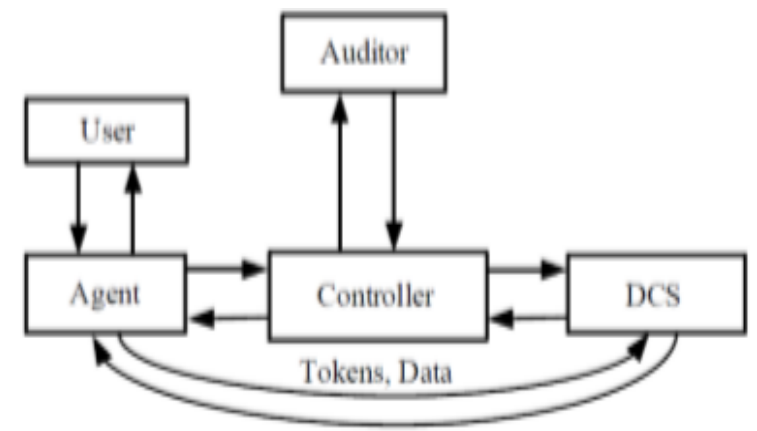

Fig. 4, Methods of encrypted data handling

Proposed a system of storage and generation of metadata based on checksums of information and time is introduced. Proposed method derived the set of methods of encrypted data handling in DCS that develop consists of procedures of saving, receiving and deleting data from DCS. All operations with encrypted data carried out in cloud computing should be performed without decryption. It is essential to store encryption keys beyond cloud computing.

\section{4.5 ENHANCED RSA ALGORITHM WITH VARYING KEY SIZES FOR DATA SECURITY}

The main technique utilized in cryptography will be encryption. This technique conserves integrity \& confidentiality of information. The confidentiality characteristic will be information constantly produced available to approved gatherings. The integrity is not permitting unapproved gathering to change the information that will be saved by client. Numerous clients are using this access control to their data is confidential.

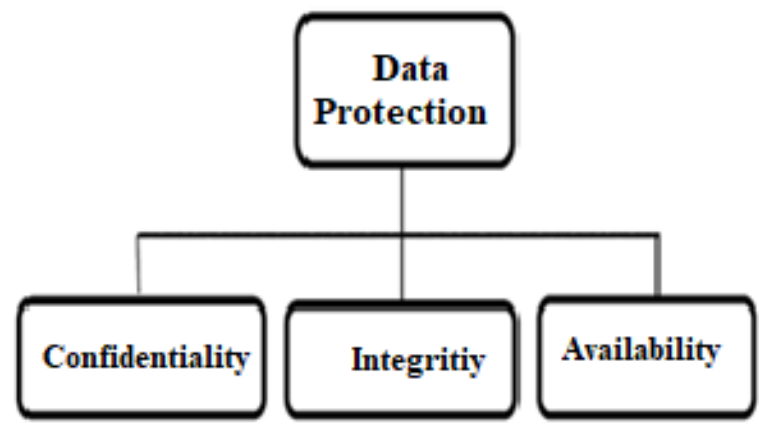

Fig. 5, Data protection method

The encryption may be completed by utilizing 1 popular asymmetric or symmetric key method like DES, AES, Blowfish, DES, RSA, \& triple DES and so on; RSA method is a asymmetric key method utilizing 2 diverse keys for decryption \& encryption procedures. 


\section{Improved Data Security for Storing and Authenticating in Cloud using ERSA Algorithm}

The size of key might be varied to make encryption procedure will be strong. Therefore, it will be critical for attackers to interrupt the information. Expanding the size of key consistently enhances the time for decryption \& encryption procedure. The suggested method lessens the time for decryption \& encryption procedures by separating the document under pieces $\&$ increases the quality of method by expanding the size of key. This quality paves the path to store the information in cloud towards the clients without any trouble.

\section{ANALYSIS AND DISCUSSION}

The auditing protocol support dynamic data operations, which is efficient and has been analyzed to be secure in the random oracle model. Proposed auditing protocol provides the support for bidirectional authentication and statistical analysis [1].

The access control is the main significant dimension to ensure CC safety. The mutual trust among cloud service nodes \& clients have ensured by trust method. The trust clients are access to cloud, and concurrently clients might choose the most credible service nodes of cloud [2].

A new method for query authentication outcomes in cloud based storage framework with help for numerous sources, which attains constant computation \& communication overheads irrespective size of data, or the several sources [3]. Whereas utilizing CC, DCS in specific, there arises an extent of topical problems associated to integrity \& confidentiality of data in procedure of transmission \& storage. Few services outside the cloud for data management, authentication, \& storage of metadata to remove the probability of acquiring unauthorized access of information, \& utilization of metadata to execute integrity control [4].

\section{Proposed Methodology}

First, Proposed Methodology proposes schemes which will helps to enhance security and authentication in cloud services. Proposed method introduce encryption and description scheme which eventually improves authentication process. First user login if user exists otherwise first user registration by using registration form. After the successful completion of login process then user can himself get the authority for storing the data into the cloud. Authorized user can select the two random picture or image from the gallery then form the one new image from these two images and this one new image is called as image fusion because this new image is a combination of these two images. Image fusion uses the numbers of XOR keys then user can select the random XOR key number. From these keys one strong password is generated and using this password, the user can select required data those are placed into the cloud. So that user can select maximum data for storing into the cloud and also achieve the security for stored data.

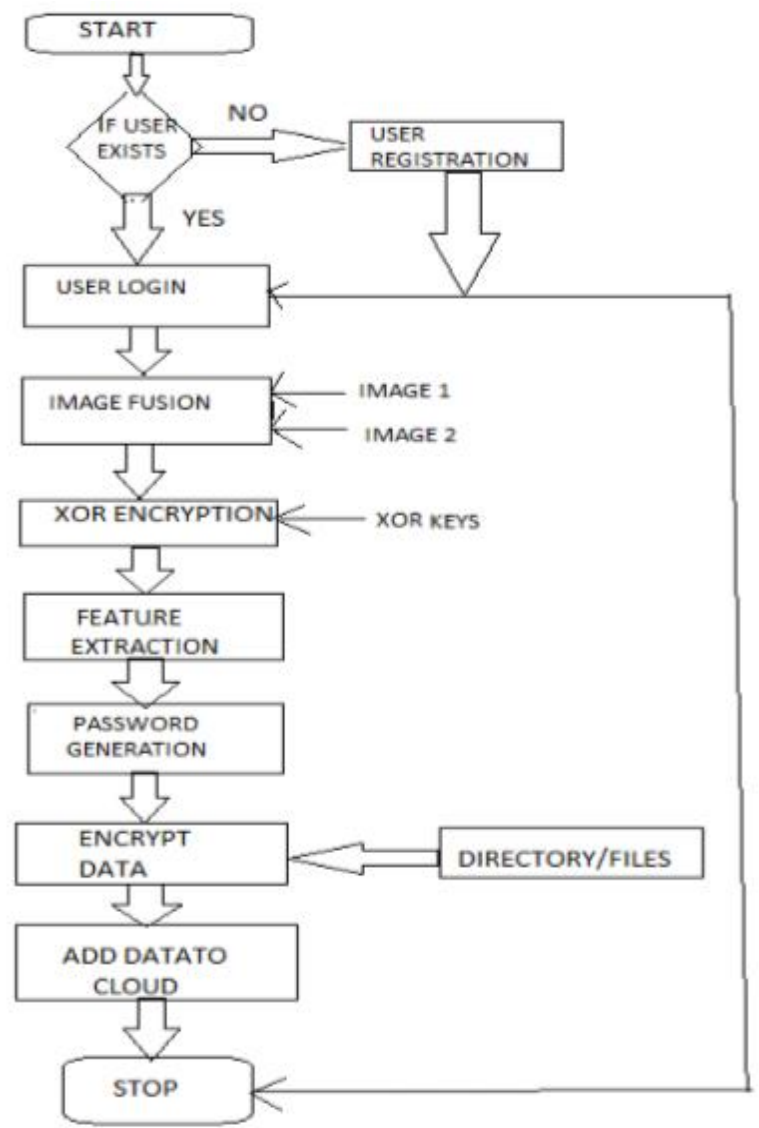

Figure.6. Data Encryption

\section{Outcome AND Possible Result}

This paper proposes a development concerning decryption \&encryption time. The decryption \& encryption time for secure RSA, high speed method \& the suggested algorithm ERSA are taken into consideration for better performance. It is extremely scalable in terms of communication \&computation overheads irrespective of data size, or numerous sources. Thus, overall, the proposed scheme achieves superior scalability and efficiency.

\section{Conclusion}

This manuscript concentrated on speed is still improved in suggested method ERSA by separating the document into numerous blocks. Apart from incrementing the speed, the execution of ERSA method makes the increments the security strength. The outcome represents the decrease in decryption \& encryption time as stated by ERSA than secure RSA \& high speed.

\section{FUTURE SCOPE}

In this paper suggested method ensures the integrity \&authenticity of choosing the query outcomes. In future plan is to prolong this mechanism to offer complete assurances that permit the customer to prove the cloud, returns each document, which satisfies the situation of query, help the diverse kinds of queries. 


\section{REFERENCES}

1. M. Armbrust, A. Fox, R. Griffith et al., \|A view of cloud computing,\| Communications of the ACM, 2013, vol.53, no.4, pp.50-58.

2. Guoyuan Lin, YuyuBie, Min Lei. Trust Based Access Control Policy in Multi-Domain of Cloud Computing. IEEE Journal 2013, pp.1357-1365.

3. J.YuanandS.Yu,-Efficientpublicintegritycheckingforclouddatashar ingwithmulti-user modification,l in Proceedings of INFOCOM, IEEE Conference on Computer Communications, Toronto, Canada, April 27 - May 2. IEEE, 2014, pp.2121-2129.

4. Boyen X. General Ad Hoc Encryption from Exponent Inversion IBE. EUROCRYPT 2015. V. 4515. P. 394-411.

5. VishwanathS.Mahalle,AniketK.Shahade,-Enhancingthedatasecurityi nCloudby mplementing Hybrid Encryption Algorithmll, IEEE, 2016, pp.146-149. 